\title{
Hypoglycemic agents and potential anti-inflammatory activity
}

\author{
This article was published in the following Dove Press journal: \\ Journal of Inflammation Research \\ II April 2016 \\ Number of times this article has been viewed
}

\author{
Vishal Kothari' \\ John A Galdo \\ Suresh T Mathews ${ }^{3}$ \\ 'Department of Nutrition and \\ Dietetics, Boshell Diabetes and \\ Metabolic Diseases Research \\ Program, Auburn University, \\ Auburn, ${ }^{2}$ Department of Pharmacy \\ Practice, ${ }^{3}$ Department of Nutrition \\ and Dietetics, Samford University, \\ Birmingham, AL, USA
}

\begin{abstract}
Current literature shows an association of diabetes and secondary complications with chronic inflammation. Evidence of these immunological changes include altered levels of cytokines and chemokines, changes in the numbers and activation states of various leukocyte populations, apoptosis, and fibrosis during diabetes. Therefore, treatment of diabetes and its complications may include pharmacological strategies to reduce inflammation. Apart from anti-inflammatory drugs, various hypoglycemic agents have also been found to reduce inflammation that could contribute to improved outcomes. Extensive studies have been carried out with thiazolidinediones (peroxisome proliferator-activated receptor- $\gamma$ agonist), dipeptidyl peptidase- 4 inhibitors, and metformin (AMP-activated protein kinase activator) with each of these classes of compounds showing moderate-to-strong anti-inflammatory action. Sulfonylureas and alpha glucosidase inhibitors appeared to exert modest effects, while the injectable agents, insulin and glucagon-like peptide-1 receptor agonists, may improve secondary complications due to their anti-inflammatory potential. Currently, there is a lack of clinical data on anti-inflammatory effects of sodium-glucose cotransporter type 2 inhibitors. Nevertheless, for all these glucoselowering agents, it is essential to distinguish between anti-inflammatory effects resulting from better glucose control and effects related to intrinsic anti-inflammatory actions of the pharmacological class of compounds.
\end{abstract}

Keywords: diabetes, inflammation, insulin, metformin, thiazolidinedione, gliptin

\section{Introduction}

Diabetes mellitus (DM) is a global health problem with a prevalence of $>382$ million human cases, and the incidence is expected to increase to 592 million by 2035 . According to the Centers for Disease Control, present trends of diabetes incidence suggest one in three Americans will be diagnosed with diabetes by the year 2050. ${ }^{1}$ The vast majority of diabetes patients (90\%-95\%) suffer from type 2 DM (T2DM), whereas type $1 \mathrm{DM}$ accounts for only 5\%-10\%. Despite the prevalence of DM and the dramatic socioeconomic and health burden, the etiology of T2DM is not fully understood. The underlying metabolic cause of T2DM can be attributed to an impairment of insulinmediated glucose disposal (insulin resistance) and a progressive defect in insulin secretion by pancreatic $\beta$-cells. ${ }^{2}$ Type $1 \mathrm{DM}$, on the other hand, is a multifactorial, organ-specific autoimmune disease, in genetically susceptible individuals, characterized by a selective and progressive loss of insulin-producing $\beta$-cells. ${ }^{3}$

Advancements in the understanding of DM have assisted in the development of numerous clinical guidelines that help shape the treatment of DM. ${ }^{4,5}$ Recently, studies have changed the perspective of DM from a metabolic disease to an inflammatory 
condition. Current literature recognizes that chronic lowgrade subclinical inflammation is a part of insulin resistance and strongly related to the features of metabolic syndrome. ${ }^{6-8}$ In addition, inflammatory processes are also involved in the microvascular complications of diabetes including diabetic nephropathy and retinopathy. ${ }^{9}$ Inflammatory factors, which play a critical role in the development of atherothrombosis, are often found to be at elevated levels in patients suffering from diabetes. Subsequently, correcting inflammation may be beneficial to stop, impede, and improve diabetes and its associated complications. For the management of T2DM, many approaches, which can target inflammation, have been investigated, but no approach has emerged for use in clinical practice., ${ }^{4,5}$ The current pharmacotherapy of T2DM is derived from the ominous octet concept described by DeFronzo.,10,11 The progressive nature of T2DM warrants an adaptive pharmacotherapy plan, which depends on a combination of medications, nutrition management, and exercise. ${ }^{12}$ Among the currently used hypoglycemic agents, some exert anti-inflammatory activity, which may help to improve clinical outcomes. Additionally, hypoglycemic drugs could potentiate the anti-inflammatory activity of other drugs used in combination therapy.

Since the prevention and improvement of T2DM conditions may not merely lie in strict glucose control, the antiinflammatory characteristics of antidiabetic agents and the inflammatory process that can evoke T2DM are reviewed here, and summarized in Figure 1. To identify such reports, an extensive search of the literature was performed using PubMed, with the terms "inflammation" and "anti-inflam- matory" combined with the names of each pharmacological class of glucose-lowering agents (biguanides, sulfonylureas, meglitinides, $\alpha$-glucosidase inhibitors, thiazolidinediones [TZDs], dipeptidyl peptidase-4 [DPP-4] inhibitors, sodiumglucose cotransporter type 2 [SGLT2] inhibitors, glucagonlike peptide-1 receptor agonists [GLP-1RAs], and insulin) or the generic names of any commercially available medications within each class. In addition, the anti-inflammatory activity of established hypoglycemic bioactive compounds is briefly reviewed.

\section{Interdependence of inflammation and diabetes}

Shoelson et al have traced the historical reports of the association of inflammation with glucose metabolism to the early 1800 s, citing the effect of high-dose salicylates to lower glycosuria in individuals with diabetes. ${ }^{13}$ It was only in 1990s that Hotamisligil et al established tumor necrosis factor alpha (TNF- $\alpha$ ) as a link between obesity, inflammation, and insulin resistance. ${ }^{14}$ Subsequent studies have shown an association of inflammation with diabetes in animal models and in humans. ${ }^{15,16}$ Cross-sectional and prospective studies have shown increased concentrations of markers of the acute phase response (including $\mathrm{C}$-reactive protein [CRP], serum amyloid-A, and sialic acid), white blood cells, plasma levels of coagulation factors (fibrinogen and plasminogen activator inhibitor 1 ), pro-inflammatory cytokines (TNF- $\alpha$, interleukin [IL]-1 $\beta$ and IL-6), and chemokines in patients with obesity and T2DM. ${ }^{17}$ In addition, inflammation in adipose tissue, liver, muscle, and pancreas has been clearly estab-

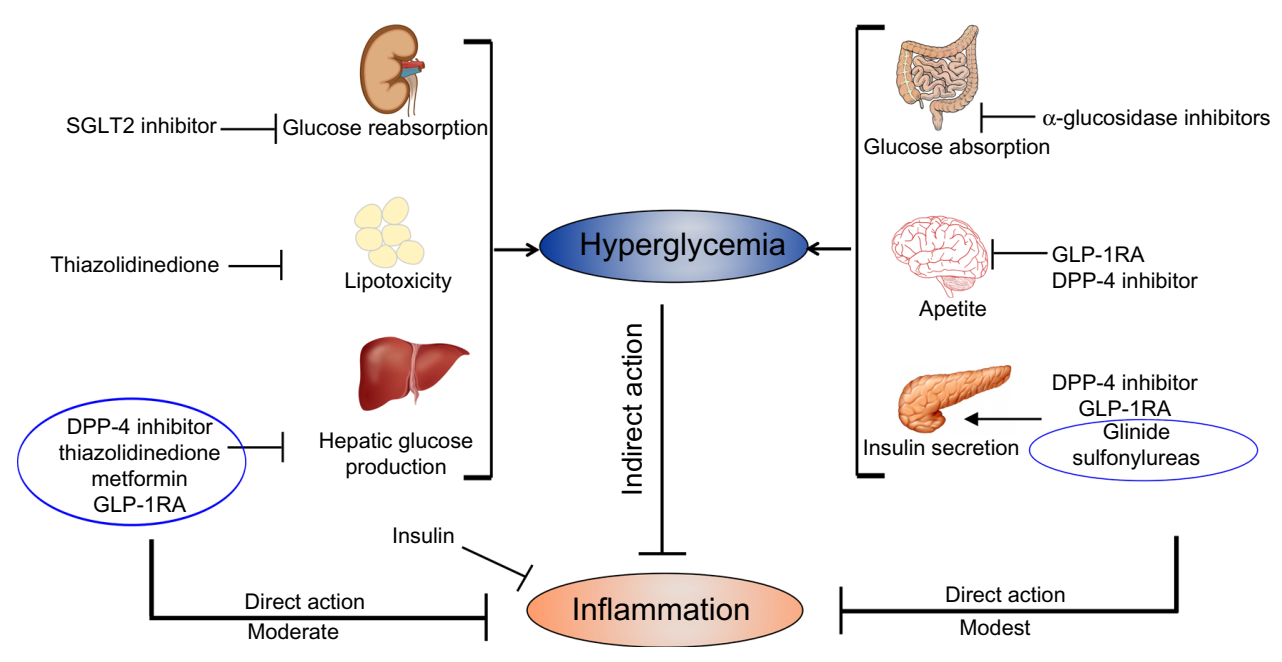

Figure I Impact of antidiabetic agents on inflammation.

Notes: Antidiabetic drugs indirectly decrease inflammation by controlling hyperglycemia by acting on respective target tissues. Hypoglycemic agents also have direct antiinflammatory activity independent of their effect on hyperglycemia.

Abbreviations: SGLT2, sodium-glucose cotransporter type 2; GLP-IRA, glucagon like peptide-I receptor agonist; DPP-4, dipeptidyl peptidase-4. 
lished in patients with obesity and T2DM. An infiltration of macrophages into these tissues is observed in animal models of obesity and diabetes, and in human obesity, metabolic syndrome, and T2DM. ${ }^{18}$ Additionally, a phenotypic switch from the anti-inflammatory (M2 macrophages) to proinflammatory phenotype (M1 macrophages) has been shown to be associated with insulin resistance, both in mice and humans. ${ }^{19}$ Activated macrophages infiltrate adipose tissue, pancreatic islets, liver, and skeletal muscle and stimulate the production of pro-inflammatory cytokines, including TNF- $\alpha$, IL-6, and IL-1 $\beta$. The pro-inflammatory cytokines act in an autocrine and paracrine manner to promote insulin resistance by interfering with insulin signaling in peripheral tissues through activation of the c-jun $\mathrm{N}$-terminal kinase (JNK) and nuclear factor-kappa B (NF-אB) pathways. ${ }^{19}$ In the pancreatic islets, local inflammation can reduce insulin secretion and trigger $\beta$-cell apoptosis, leading to a decrease in islet mass, which are all critical events in the progression of T2DM. ${ }^{20}$ Furthermore, the adaptive immune system-like increase in cytotoxic $\mathrm{CD}^{+}$and imbalance between proinflammatory and anti-inflammatory $\mathrm{CD}^{+}$cells may also contribute to diabetes. ${ }^{21}$ Although subclinical inflammation is important in the pathogenesis of T2DM, the events initiating this inflammatory process remain unclear and could involve different but synergic mechanisms, leading to the activation of NF-KB and JNK pathways, cytokines, and chemokines release and recruitment of immune cells.

\section{Anti-inflammatory effect of injectable hypoglycemic agents Insulin}

Insulin, discovered in 1921, is a key metabolic hormone with profound effects on glucose and lipid metabolism. ${ }^{22}$ The pleiotropic effects of insulin including anti-inflammatory, antithrombotic, and antioxidant properties suggest that insulin suppresses the inflammatory process not only by controlling hyperglycemia but also by directly modifying key inflammatory molecules involved in pathophysiology of diabetes. ${ }^{25}$ Insulin induces expression of endothelial nitric oxide (NO) synthase through the activation of phosphoinositide-3kinase (PI3K) and Akt kinase (protein kinase B), resulting in the generation of NO that can increase blood flow and inhibit platelet aggregation. ${ }^{23}$ In vitro and in vivo studies have further confirmed that insulin suppresses three important inflammatory mediators: intercellular cell adhesion molecular-1; monocyte chemoattractant protein (MCP)-1 expression; and $\mathrm{NF}-\kappa \mathrm{B}$ binding in human aortic endothelial cells. ${ }^{24}$ Insulin has also been shown to suppress vascular endothelial growth factor, a cytokine that is involved in the pathogenesis of diabetic retinopathy and myocardial infarction. ${ }^{23}$ Finally, insulin suppresses toll-like receptor 4 (TLR-4), a pattern recognition receptor of endotoxin involved in tissue inflammation, and has been shown to mediate diet-induced obesity, insulin resistance, vascular inflammation, and atherogenesis. ${ }^{26}$ Though these studies have not provided a specific mechanism involved in the protective effects of insulin, they suggest that insulin can suppress inflammation by modulating vascular permeability and cytokine production.

Outcomes of insulin pharmacotherapy lend support to its anti-inflammatory role in DM. Results from the United Kingdom Prospective Diabetes Study showed that aggressive glycemic control significantly reduced the risk of any microvascular end point compared to conventional treatment. ${ }^{27}$ However, there was no significant difference in macrovascular risk among those treated intensively with insulin during the study period. ${ }^{28}$ In the ACCORD trial, the findings of higher mortality in the intensive-therapy group led to the discontinuation of intensive therapy after a mean of 3.5 years of follow-up. ${ }^{29}$ Another randomized parallelgroup study in patients with newly diagnosed T2DM showed that intensive, short-term insulin therapy improved $\beta$-cell function. ${ }^{28}$ However, suppressive effects on inflammatory biomarker high-sensitive CRP (hsCRP) in T2DM have been unequivocal, and this is seen in a randomized, parallel-group study in patients with newly diagnosed T2DM, which suggests that insulin can reduce hsCRP and IL-6, independent of the reduction in blood glucose. ${ }^{28}$ Alternatively, Da Silva et al observed that subcutaneous insulin therapy does not interfere with venous endothelial function in T2DM, given stable glycemic and blood pressure control. ${ }^{31}$ Treatment of insulin in patients with poorly controlled T2DM reduced serum hsCRP levels, without affecting plasma fibrinogen or serum MCP-1 levels. ${ }^{30}$ However, in young obese Latino patients with T2DM, improvement of glycemic control with insulin monotherapy was not associated with a parallel improvement in markers of vascular inflammation (hsCRP). ${ }^{32}$ Also, in the LANCET trial, treatment with insulin compared with a placebo or metformin did not reduce inflammatory biomarker levels despite improving glucose control. ${ }^{33}$

Currently, the lack of large-scale randomized doubleblind control trials to study anti-inflammatory effects of insulin is a major limitation in drawing clinical conclusions. Secondly, insulin has a major drawback in terms of inducing weight gain. This increase in fat mass can bring distinct morphological changes including adipocyte enlargement and macrophage influx leading to a more pronounced 
inflammatory status reflected by an increased secretion of pro-inflammatory mediators and a reduction in secretion of the insulin-sensitizing protein adiponectin (ADN). Therefore, the systemic anti-inflammatory effects of insulin may be counteracted by the pro-inflammatory changes associated with an increased fat mass, which is reinforced by a study from Jansen et al where patients characterized by a pronounced insulin-associated weight gain had an influx of macrophages into the adipose tissue and it was accompanied by a more pronounced inflammatory status. ${ }^{34} \mathrm{~A}$ variety of insulin analogs and administration techniques are available which may lower the risk of hypoglycemia and result in less weight gain, thus providing the tools to overcome barriers commonly associated with insulin therapy. ${ }^{35}$

\section{Glucagon-like peptide analogs and agonists}

Glucagon-like peptide-1 (GLP-1), a gut incretin hormone secreted from Langerhans cells in the intestine in response to oral nutrient intake, has been considered an attractive agent for the management of T2DM. GLP-1RAs and other analogs have been shown to activate GLP-1 receptor to increase intracellular cAMP in pancreatic acinar cells to stimulate insulin secretion while suppressing glucagon secretion and functions identically to GLP-1. ${ }^{36}$ This class of medications now has a role in the treatment of obesity and protection against cardiovascular and neurological diseases partly due to its anti-inflammatory potential. ${ }^{37}$ Additionally, GLP-1RAs decrease waist circumference, fat content, and intrahepatic lipids in patients with nonalcoholic fatty liver diseases and T2DM. ${ }^{38}$ These medications have anti-inflammatory effects in different cell types including human umbilical vein endothelial cells, glomerular endothelial cells, monocytes, and macrophages. ${ }^{39}$ Exendin-4 was found to reduce lipopolysaccharide (LPS)-induced inflammation in 3T3-L1 adipocytes and adipose tissue macrophage (ATM). ${ }^{36}$ The GLP-1RAs improve the inflammatory response by reducing the release of inflammatory cytokines, by improving inflammatory pathways, and increasing the anti-inflammatory adipokine, ADN. In addition, treatment with liraglutide or exenetide was found to decrease CRP in patients with T2DM. ${ }^{40}$ The expression and production of IL-6, TNF- $\alpha$, and MCP-1 were significantly reduced in adipose tissue of recombinant adenovirus-producing GLP-1-treated obese (ob/ob) mice. ${ }^{40}$ Various in vitro and in vivo studies suggest that GLP-1RAs show anti-inflammatory potential by inhibition of I $\kappa \mathrm{B}$ kinase beta/NF- $\kappa \mathrm{B}$ pathway and the JNK pathway, and the mechanism of anti-inflammatory action of GLP-1RAs involved modulation of the canonical Wnt signaling and other inflammatory pathways. ${ }^{36}$

\section{Anti-inflammatory effect of oral hypoglycemic agents Biguanides (metformin)}

Metformin and other biguanides are derived from French lilac (Galega officinalis), and since the 1950s, metformin is the first choice in the oral treatment of T2DM. ${ }^{4,5,41}$ Several mechanisms of the glucose-lowering effect of metformin have been proposed including the generally accepted activation of AMP-activated protein kinase (AMPK), a cellular energy sensor activated under metabolic stress. Metformin not only improves hyperglycemia and insulin resistance but also has been shown to have anti-inflammatory, anticancer, and antiaging effects and to improve other cardiovascular risk factors, such as an overweight state or obesity, atherogenic dyslipidemia, blood pressure, procoagulant state, and carotid intimamedia thickness. ${ }^{41}$ Metformin activates AMPK to inhibit NF- $\kappa \mathrm{B}$ via PI3K-Akt pathway in human vascular smooth muscle cells for anti-inflammatory action. ${ }^{42}$ Metformin may also reduce the production of NO, prostaglandin E2, and proinflammatory cytokines (IL-1 $\beta$, IL-6, and TNF- $\alpha$ ) through inhibition of NF- $\mathrm{BB}$ activation in macrophages. ${ }^{42}$ Recently, it has been shown that AMPK interacts with tumor suppressor gene PTEN, to antagonize PI3K, and affect cell survival, growth, and proliferation. ${ }^{43}$ Metformin can also regulate inflammation through SIRT1/LKB1/AMPK pathway and inhibit cellular metabolic memory through suppression of reactive oxygen species/Poly ADP-ribose polymerase (PARP) signaling. ${ }^{44}$ In addition, metformin can exhibit anti-inflammatory action through inhibition of advanced glycation end products which promote inflammation and reactive oxygen species. ${ }^{45}$ Moreover, metformin exhibits anti-inflammatory action indirectly by controlling hyperglycemia, weight gain, and lipid profile resulting in a favorable effect on chronic inflammation and atherosclerosis. ${ }^{41}$

In the Diabetes Prevention Program, treatment with metformin in patients with impaired glucose tolerance (IGT) for 12 months decreased CRP levels as compared to placebo. ${ }^{46}$ Krysiak and Okopien showed that patients with IGT treated with metformin reduced release of various pro-inflammatory cytokines from monocyte and lymphocytes. ${ }^{47,48}$ In the BARI $2 \mathrm{D}$ trial, treatment of metformin in patients with T2DM and coronary artery disease showed anti-inflammatory effects as indicated by reduction in plasma insulin, plasminogen activator inhibitor type 1 antigen, CRP, and fibrinogen levels. ${ }^{49}$ However, in the LANCET trial, treatment of metformin for 
14 weeks failed to decrease CRP or other inflammatory biomarkers in patients with T2DM. ${ }^{33}$ Conversely, Derosa et al reported that the combination of metformin and exenatide reduced inflammatory markers such as vaspin, chemerin, and resistin in patients with T2DM compared with metformin plus placebo. ${ }^{50}$ Thus, the anti-inflammatory effect of metformin remains unclear and may be an indirect effect mediated through the improvement of insulin sensitivity and hyperglycemia.

\section{Sulfonylureas}

Sulfonylureas target the ATP-sensitive potassium $\left(\mathrm{K}_{\text {ATP }}\right)$ channel to stimulate insulin secretion from pancreatic $\beta$-cells and are widely used in the treatment of T2DM.,5,51 The $\mathrm{K}_{\mathrm{ATP}}$ channels in monocytes/macrophages are upregulated and stimulate inflammatory reactions mediated by MAPKs/NF- $\mathrm{KB}$ pathways, while glibenclamide, a generic sulfonylurea (US generic glyburide), rescues this progression. ${ }^{52}$ Cai et al found that glibenclamide could attenuate LPS-induced myocardial injury in diabetic mice, possibly through inhibiting inflammation. ${ }^{53} \mathrm{Mu}-\mathrm{Huo}$ et al showed that in a polymicrobial sepsis animal model, glibenclamide pretreatment attenuates the acute lung injury by inhibiting the inflammatory responses and oxidative stress. ${ }^{54}$ Inhibition of sulfonylurea receptor 1 by glibenclamide ameliorates several pathologic effects associated with inflammation that lead to cortical dysfunction after subarachnoid hemorrhage. ${ }^{55}$ In addition, glibenclamide reduces pro-inflammatory cytokine production by neutrophils in patients with diabetes in response to bacterial infection. ${ }^{56}$ Treatment of glyburide in patients with T2DM increases survival rate associated with the change in anti-inflammatory-related genes. Mavridis et al reported that $\mathrm{T} 2 \mathrm{DM}$ patients treated with insulin presented with a low-grade inflammation, while the levels of inflammatory cytokines in the sulfonylurea arm were significantly lower than insulin-treated. ${ }^{57}$ Studies on sulfonylureas provide evidence for safety in patients with diabetes combined with asthma by downregulation of allergic inflammation via IL-4/ IL-13/p-STAT6/VCAM-1 signaling pathway or by inhibiting cytokine-induced eosinophil survival and activation. ${ }^{58}$

\section{Alpha-glucosidase inhibitors}

Postprandial glucose load gives a greater increase in biomarkers of systemic low-grade inflammation and endothelial dysfunction such as hsCRP, IL-6, TNF- $\alpha$, soluble intercellular adhesion molecule 1, soluble vascular cell adhesion molecule 1, E-selectin, and metalloproteinases 2 and 9 in patients with T2DM compared to healthy patients. ${ }^{59,60}$ Extensive stud- ies have been carried out on alpha-glucosidase inhibitors that suppress postprandial glucose which may mediate potential anti-inflammatory effects. Fukaya et al suggested that miglitol inhibits the gene expression of inflammatory cytokines/cytokine-like factors in peripheral leukocytes by suppressing glucose fluctuations in streptozotocin-induced hyperglycemic rats. ${ }^{61}$ Derosa et al evaluated effects of acarbose in patients with T2DM and found acarbose more effective in reducing the postoral fat-load peaks of various parameters including the insulin resistance and the inflammatory markers, after 7 months of therapy. ${ }^{62,63}$ Emoto et al studied patients with T2DM and coronary artery disease with 3 months of repeated administration of miglitol demonstrating an improvement in insulin resistance index, CRP, and flow-mediated dilatation but not with voglibose. ${ }^{64}$ However, in a randomized double-blind, placebo-controlled crossover study, reduction of glycemic load by acarbose had no effect on ADN, whole-body insulin sensitivity, or biomarkers of inflammation (MCP-1, IL-6, and IL-1 $\beta$ ). ${ }^{65}$ Similarly, comparison of pioglitazone versus voglibose by Fujitaka et al showed an improvement in hemoglobin A1c (HbA1c), fasting plasma glucose (FPG), serum ADN, and hsCRP levels and homeostatic model assessment of insulin resistance only in the pioglitazone group. ${ }^{66}$

\section{Meglitinides}

Meglitinides, or glinides, reduce postprandial blood glucose peaks and reduce $\mathrm{HbA} 1 \mathrm{c}$ levels by acting as a meal-time insulin secretagogue similar to the sulfonylureas. Although novel anti-inflammatory effects of repaglinide in nondiabetic animals were established, the high doses required for an efficacious effect would make this application impractical in the clinic. ${ }^{67}$ Repaglinide was studied in two different models of delay-type hyperreactivity response induced by sheep red blood cells and 2,5'-dinitrofluorobenzene, and in two different rodent models of LPS challenge. Repaglinide efficaciously downregulated the resulting ear swelling response with sheep red blood cells and decreased serum TNF- $\alpha$ level and bronchial alveolar lavage fluid MCP-1 levels in LPSchallenged animals. ${ }^{67}$ In addition, change of treatment from glimepiride to repaglinide in Japanese patients with T2DM reduced the levels of plasminogen activator inhibitor-1, hsCRP, and urinary 8-hydroxydeoxyguanosine. ${ }^{68}$ Similarly, a study by Assaloni et al showed that controlling postprandial hyperglycemia with meglitinide significantly improves the cluster of oxidative stress (reduces nitrotyrosine, malondialdehyde, and oxidized low-density lipoprotein levels) and inflammation markers (decreases IL-6, IL-18, and TNF- $\alpha$ ) 
that are increased in the postprandial state in patients with diabetes. ${ }^{69}$ Alternatively, repaglinide in diabetic rabbits exerted antioxidant properties without affecting serum IL-6 levels. ${ }^{70}$ However, in a study in nonobese patients with T2DM, metformin was more effective in reducing selected biomarkers reflecting inflammation and endothelial dysfunction compared with repaglinide despite similar glycemic levels between treatments. ${ }^{71}$ In the NAVIGATOR trial, treatment with nateglinide for 5 years did not reduce the incidence of diabetes or the co-primary composite cardiovascular outcomes in patients with IGT and established cardiovascular disease or cardiovascular risk factors. ${ }^{72}$ Effect of nateglinide versus acarbose in 160 Chinese patients with T2DM has been studied, and no significant effect on hsCRP was found in either group despite similar effects in controlling postprandial glucose and lipid metabolism. ${ }^{73}$

\section{Thiazolidinedione}

Peroxisome proliferator-activated receptors (PPARs), mainly PPAR- $\gamma$, have potential implications in molecular pathways of insulin resistance, T2DM, and atherosclerosis. ${ }^{74}$ Rosiglitazone and pioglitazone, also known as TZDs, are selective agonists of nuclear transcription factor PPAR- $\gamma$. In vitro data in animal models and some human studies suggested that TZDs may not only regulate metabolic processes but also limit inflammatory responses. ${ }^{74}$ Specifically, PPAR- $\gamma$ is expressed predominantly in adipose tissue and shown to reduce inflammatory markers in visceral adipose tissue (VAT), steatotic liver, atherosclerotic plaques, and circulating plasma. ${ }^{75}$ Moreover, in vitro results demonstrate that the anti-inflammatory activity of TZDs is a result, at least in part, of their ability to activate glucocorticoid nuclear translocation independently from PPAR $-\gamma^{75}$

Treatment of pioglitazone in patients with T2DM reduced ATM content and activity, and was associated with a decrease in inflammatory markers in macrophage, neutrophils, and dendritic cells. ${ }^{76}$ In this study, hepatic and peripheral insulin sensitivity was improved, indicating an association between adipose macrophage content and systemic insulin resistance in humans. ${ }^{76}$ Patients with insulin resistance had a decreased total adipose macrophage number, with a decrease in M1 macrophages and an increase in M2 macrophages with pioglitazone treatment. ${ }^{77}$ Pioglitazone improved ADN levels significantly and increased adipocyte cell surface but failed to reduce ATM number and pro-inflammatory $\mathrm{CD} 16^{+}$peripheral monocyte numbers in obese individuals despite improvement in insulin sensitivity. ${ }^{78}$ Another study in obese subjects had a decrease in insulin resistance and several adipose inflammatory parameters (IL-6, IL-1 $\beta$, and inducible nitric oxide synthase) with pioglitazone relative to placebo. ${ }^{79}$ Additionally, adipose tissue from pioglitazone-treated obese subjects demonstrated reduced infiltration of macrophages, dendritic cells, and neutrophils as well as increased expression of factors associated with fat "browning" (PGC-1 $\alpha$ and UCP-1). ${ }^{79}$ Finally, a study by Kodama et al indicated that pioglitazone, but not glimepiride, decreased the visceral fat volume and its metabolic activity in patients with IGT or T2DM. ${ }^{80}$ Recently, a unique population of VAT-resident regulatory $\mathrm{T}$ cells was implicated in control of the inflammatory state of adipose tissue, and thereby, insulin sensitivity. PPAR- $\gamma$ expression by VAT-resident regulatory $\mathrm{T}$ cells was necessary for complete restoration of insulin sensitivity in obese mice by pioglitazone. ${ }^{81}$ Meta-analysis of randomized, placebo-controlled clinical trials showed that TZDs were significantly better than placebo in improving ballooning degeneration, lobular inflammation, and steatosis in patients with nonalcoholic steatohepatitis. However, evidence suggest that long-term treatment with these agents offers no additional histological benefit parallel to metabolic improvement. ${ }^{82,83}$

Mizoguchi et al showed that pioglitazone, but not glimepiride, decreased atherosclerotic plaque inflammation despite reduction of FPG and $\mathrm{HbA} 1 \mathrm{c}$ values. Additionally, pioglitazone significantly increased high-density lipoprotein cholesterol level and decreased hsCRP, whereas hsCRP was increased by glimepiride. ${ }^{85,86}$ In another study, compared with placebo, rosiglitazone reduced inflammatory markers, ubiquitin, proteasome 20S activity, nitrotyrosine, and superoxide anion production and increased collagen content in carotid plaque. The inhibition of ubiquitin-proteasome activity in atherosclerotic lesions of patients with T2DM by rosiglitazone was associated with morphological and compositional characteristics of a potential stable plaque phenotype, possibly by downregulating NF- $\mathrm{BB}-$ mediated inflammatory pathways. ${ }^{87}$

In many studies, measuring circulating markers has assessed the impact of intervention with TZDs on inflammation, atherosclerotic plaque, and cardiovascular outcomes. In patients with T2DM and coronary artery disease, treatment with pioglitazone resulted in a significantly lower rate of progression of coronary atherosclerosis compared with glimepiride. This is associated with decrease in hsCRP levels, increase in high-density lipoprotein level, and reduction in triglyceride levels by pioglitazone, but not with glimepiride. ${ }^{88}$ The metaanalysis study on the effects of TZD therapy in patients with T2DM reported significant reductions in the majority of plasma cytokines, such as hsCRP, MCP-1, fibrinogen, and E-selectin, compared with those of the placebo group among patients with T2DM. ${ }^{89}$ Updated meta-analysis indicated that 
both rosiglitazone and pioglitazone significantly decreased serum CRP levels in both DM and non-DM patients. ${ }^{90}$ This is confirmed by an observational IRIS V study of a nonselected patient population under daily routine conditions. The results show that pioglitazone treatment improved chronic vascular inflammation, which may be associated with reduced cardiovascular risk as monitored by the biomarker CRP. ${ }^{91}$

Nitta et al compared the effect of pioglitazone with glimepiride on coronary arterial inflammation with serial 18F-fluorodeoxyglucose-positron emission tomography combined with computed tomography angiography. In this study, pioglitazone, but not glimepiride, attenuated left main trunk inflammation as measured by $18 \mathrm{~F}$-fluorodeoxyglucose uptake in patients with IGT or T2DM. However, glimepiride and pioglitazone decreased clinical parameters, including FPG and HbA1c. ${ }^{85,86}$ These studies suggest that pioglitazone may protect against cardiac events in patients with IGT or T2DM in a glucose-lowering independent manner by suppressing coronary inflammation. The PROactive study showed that pioglitazone nonsignificantly reduces the risk of all-cause mortality, myocardial infarction, stroke, acute coronary syndrome, leg amputation, coronary revascularization, or revascularization of the leg, which is further supported by BARI 2D trial which compared the effect of insulin sensitization (IS) and insulin provision (IP) treatment strategies on adipokines associated with cardiovascular disease in patients with T2DM and coronary artery disease. ${ }^{49,92}$ The BARI $2 \mathrm{D}$ trial shows that IS and IP treatment strategies exerted divergent effects on adipokine and inflammatory profile, and IS treatment strategy-induced changes may be more favorable than the IP treatment strategy regarding cardiovascular pathophysiology. ${ }^{49}$

\section{DPP-4 inhibitors}

DPP-4 inhibitors, commonly referred to as gliptins, improve glucose metabolism through inhibition of degradation of endogenous GLP-1, which causes the elevation of GLP-1 receptor signaling, leading to increased insulin secretion and suppressed glucagon secretion in the pancreas. ${ }^{93}$ Similar to GLP-1RAs, several studies have shown that DPP-4 inhibitors offer additional clinical advantages including beneficial effects on the cardiovascular system without risk of hypoglycemia and weight gain. ${ }^{94}$ Therefore, incretin-based therapies might open new perspectives in the management of T2DM by providing some cardiovascular protection in patients with T2DM. DPP-4 inhibitors can exert antiatherogenic effects which include lowered systolic blood pressure, improved postprandial lipid parameters, reduced silent inflammation, reduced oxidative stress, and improved endothelial dysfunction. ${ }^{95}$ Several experimental studies report direct potential beneficial effects of DPP-4 inhibitors on all microvascular diabetes-related complications including microangiopathy. ${ }^{96}$ Animal studies have shown that the effect of DPP-4 inhibition on atherosclerosis and inflammation is via inhibition of monocyte activation and chemotaxis. Treatment with linagliptin remarkably suppressed cardiac fibrosis in an experimental model of autoimmune myocarditis mice and was associated with reduced inflammatory cytokine (IL-2, TNF- $\alpha$, IL-1 $\beta$, and IL-6) gene expression. ${ }^{97}$ Linagliptin treatment was also found to attenuate inflammation and accelerate epithelialization in wounds of diabetic ob/ob mice..$^{98}$ Consistent with linagliptin, in vitro studies have shown that alogliptin inhibited TLR-4-mediated upregulation of IL-1 $\beta$, IL-6, and other pro-inflammatory cytokines by mononuclear cells. ${ }^{99}$ Treatment with sitagliptin suppressed oxidized lowdensity lipoprotein-induced Nucleotide-binding domain, leucine-rich repeat family, pyrin domain 3 (NLRP3), TLR-4, and IL-1 $\beta$ in human macrophages through inhibition of protein kinase 3 (PKC) activity. ${ }^{100}$ Further, in another study, anagliptin treatment prevented LPS- or palmitate-induced elevations of inflammatory cytokines in macrophages, adipocytes, and liver in mice, by suppressing NF- $\kappa B$ activation. ${ }^{101}$ In addition, treatment with sitagliptin inhibits mononuclear mRNA expression of CD26 (with known DPP-4 activity), TNF- $\alpha$, TLR-4, TLR-2, and I $\kappa$ B kinase beta, and chemokine receptor CCR-2, in T2DM patients. The suppression of CD26 expression suggests that sitagliptin may inhibit the synthesis of DPP-4 in addition to inhibiting the DPP-4 action. ${ }^{102}$ Sitagliptin significantly reduced the plasma levels of markers of low-grade inflammation (CRP, IL-6, IL-18, and secreted phospholipase-A2) and cell adhesion molecules including soluble intercellular adhesion molecule-1 and E-selectin. ${ }^{103}$ Vildagliptin was found to be superior compared to sitagliptin in reduction of oxidative stress and inflammation by blunting daily acute glucose fluctuations in patients with T2DM. ${ }^{104}$ Treatment with sitagliptin reduced inflammatory cytokines and improved the unfavorable M1/M2-like phenotypes of peripheral blood monocytes in Japanese patients with T2DM. ${ }^{105}$ In another study, sitagliptin significantly improved endothelial function and inflammatory state in patients with coronary artery disease and uncontrolled T2DM, beyond its hypoglycemic action. ${ }^{106}$ Treatment with sitagliptin plus metformin more effectively reduced the levels of resistin, vaspin, and omentin-1 than placebo plus metformin. ${ }^{50}$ However, addition of sitagliptin or metformin to pioglitazone monotherapy in patients with poorly controlled T2DM demonstrated that only metformin led to a decrease of body weight and to a faster and superior improvement of insulin resistance and 
inflammatory parameters. ${ }^{107}$ In hemodialysis patients with T2DM, linagliptin decreased levels of prostaglandin E2, IL-6, hsCRP, glycated albumin, and blood glucose which was associated with an increase in active GLP-1. ${ }^{108}$

\section{SGLT2 inhibitors}

SGLT2 inhibition is the newest oral approach for the management of T2DM. ${ }^{4,5}$ Several compounds (dapagliflozin, canagliflozin, empagliflozin, and ipragliflozin) are already available in many countries, and others are in the late phases of development. SGLT2 inhibitors increase glucosuria, reduce hyperglycemia (without inducing hypoglycemia), promote weight loss, and exert a modest diuretic effect with blood pressure reduction. ${ }^{109}$ Ipragliflozin was found to reduce plasma and liver inflammatory markers (IL-6, TNF- $\alpha$, MCP-1, and CRP) in high-fat diet and streptozotocinnicotinamide-induced type 2 diabetic mice and in rats with streptozotocin-induced type 1 diabetes. ${ }^{110,111}$ However, no clinical data are available examining the role of SGLT2 inhibitors on markers of inflammation. ${ }^{109}$

\section{Anti-inflammatory effect of combination therapy}

Improvement in glycemic control is critical for the prevention of hyperglycemia-induced dyslipidemia, endothelial dysfunctions, vasomotor dysfunction, coagulation abnormalities, and associated complications. Since T2DM is a progressive disease, it has been accepted that combination therapy using antidiabetic agents, directed at multiple molecular targets, is more effective than monotherapy to achieve targeted blood glucose concentrations. ${ }^{10,112}$ As mentioned previously, DeFronzo has introduced the ominous octet concept for the treatment of T2DM, which advocates initial treatment with triple therapy of a biguanide, TZD, and GLP- $1 .{ }^{10}$ Also, combination therapy can increase anti-inflammatory potential of antidiabetic agents. Consequently, combination therapy is so common in the treatment of T2DM, that many medications are co-formulated as combinations, which facilitates better patient adherence. When selecting the combination treatment, it is important to consider pathogenesis of T2DM, and risk factors of cardiovascular diseases, endothelial dysfunction, and inflammation. ${ }^{112}$ Schöndorf et al carried out a multicenter, randomized, double-blinded two-arm trial in T2DM patients with combinations of metformin with pioglitazone, and metformin with glimepiride. They observed that as compared to metformin with glimepiride, the combination of metformin with pioglitazone showed an overall improvement of laboratory surrogate markers of platelet function and chronic systemic inflammation. ${ }^{113}$ Combination of sulfonylureas with metformin or TZDs offered excellent improvement in glycemic control and inflammation, which was particularly effective in the early stages of the disease, allowing maximal benefit to be obtained from the insulin secretion-promoting abilities of the sulfonylureas and the $\beta$-cell-protective effects of the TZDs. ${ }^{114}$ A potential advantage here is the combination of DPP-3 inhibitors and GLP-1RAs, which stimulate insulin secretion, suppress glucagon production, and increase or sustain GLP-1 levels in circulation. The combination of exenatide or vildagliptin plus metformin was found to be more effective than metformin alone in improving glycemic control, $\beta$-cell function, and inflammatory parameters. ${ }^{104,115}$ The novel mechanism of action of SGLT2 inhibitor therapy suggests that it has the potential to be used in combination with any of the existing glucose-lowering agents, including insulin, as they share no common mechanistic pathways. The effects of combining SGLT2 inhibitor with linagliptin treatment were assessed on pancreatic islet function and inflammation in $\mathrm{db} /$ $\mathrm{db}$ mice. The combined treatment exhibited greater effects on glycemic control, which was associated with restored islet beta/alpha-cell ratio, reduced beta-cell apoptosis, decreased expression of islet immune cell markers, and suppressed factors related to the TLR-2 pathway. ${ }^{116}$ In addition, several studies utilizing SGLT2 inhibitors plus other classes of oral antidiabetic agents are ongoing, including dapagliflozin plus metformin, dapagliflozin plus saxagliptin, canagliflozin plus metformin, and empagliflozin plus linagliptin or plus metformin. ${ }^{117}$ However, no clinical data are currently available examining the role of combination therapy of SGLT2 inhibitors with other antidiabetics on markers of inflammation.

For several patients, oral antidiabetic agents alone fail to achieve targeted glycemic control, and insulin is added to the treatment regimen. It has been suggested that after 9 years of T2DM diagnosis, $80 \%$ of patients required insulin treatment. ${ }^{112}$ Nevertheless, results from the LANCET randomized trial indicate that, compared to placebo, treatment with insulin or metformin or the combination of insulin and metformin did not reduce inflammatory biomarker levels despite improving glucose control in patients with recent-onset type 2 diabetes. ${ }^{33}$ However, new combination products are coming to market, which will combine GLP-1s and basal insulin, which could provide additional anti-inflammatory effects.

\section{Anti-inflammatory effects of hypoglycemic bioactive compounds}

Apart from synthetic hypoglycemic drugs, the plant kingdom has contributed immensely to the modern drug library, 
and several plants have been used as natural remedies for diabetes since ancient times. Bioactive constituents from medicinal plants offer a complementary approach in the management of diabetes. Grover et al reported that there are $\sim 800$ plants that exert potential antidiabetic activities. ${ }^{118}$ These bioactive compounds may act through different mechanisms, including the inhibition or stimulation of enzymatic activity and/or protein expression. The most biologically active natural compounds with proven antidiabetes activity include various polyphenols (catechins, chlorogenic acids, and flavonoids) and saponins. ${ }^{119}$ Similar to currently used hypoglycemic agents, some bioactive compounds also exert anti-inflammatory activity. Catechins were found to prevent vascular inflammation and reduce the progression of atherosclerotic lesions both in animals and humans. ${ }^{120}$ However, oral treatment of green tea catechins with vitamin $\mathrm{C}$ did not significantly reduce skin erythema, leukocyte infiltration, or eicosanoid response to ultraviolet radiation-induced inflammatory challenge in a double-blind, randomized, placebo-controlled trial. ${ }^{121}$ Chlorogenic acid was also shown to suppress cytokine-induced inflammatory process in different in vitro and in vivo studies. ${ }^{122,123}$ Flavonoids and saponins exert anti-inflammatory effects in various cell types, lowering the expression and/or function of a variety of inflammatory mediators including eicosanoids, NO, adhesion molecules, and cytokines. ${ }^{124,125}$ In addition, many bioactive compounds display significant antioxidant/radical-scavenging properties which can contribute toward anti-inflammatory effects.

\section{Conclusion}

Currently, the prevalence of obesity and T2DM has reached epidemic proportions in the USA. Since this can lead to cardio-metabolic derangements, there is a clear need to address this worrying trend. Obesity and T2DM cause an increase in inflammatory markers (hsCRP, TNF- $\alpha$, IL-6) and a decrease in anti-inflammatory factors, including ADN, leading to metabolic dysfunction. Thus, targeting inflammation is important for the management of diabetes and related disorders. Multiple studies have demonstrated an anti-inflammatory potential for various hypoglycemic drugs, which can contribute to improved clinical outcomes. Hypoglycemic agents exert their anti-inflammatory effects either by controlling hyperglycemia or directly, by acting on inflammatory pathways, independent of glucose control.

\section{Disclosure}

Kothari and Mathews report no conflicts of interest in this work. Galdo reports serving as a speaker for Novo Nordisk.

\section{References}

1. Centers for Disease Control and Prevention. Diabetes Report Card 2014. Atlanta, GA: Centers for Disease Control and Prevention, US Dept of Health and Human Services; 2015.

2. D'Adamo E, Caprio S. Type 2 diabetes in youth: epidemiology and pathophysiology. Diabetes Care. 2011;34:S161-S165.

3. Gillespie KM. Type 1 diabetes: pathogenesis and prevention. CMAJ. 2006;175:165-170.

4. Garber AJ, Abrahamson MJ, Barzilay JI, et al. AACE/ACE comprehensive diabetes management algorithm 2015. Endocr Pract. 2015;21:438-447.

5. Standards of medical care in diabetes-2015: summary of revisions. Diabetes Care. 2015;38 Suppl:S4.

6. Luft VC, Schmidt MI, Pankow JS, et al. Chronic inflammation role in the obesity-diabetes association: a case-cohort study. Diabetol Metab Syndr. 2013;5:31.

7. Esser N, Legrand-Poels S, Piette J, et al. Inflammation as a link between obesity, metabolic syndrome and type 2 diabetes. Diabetes Res Clin Pract. 2014;105:141-150.

8. Wellen KE, Hotamisligil GS. Inflammation, stress, and diabetes. J Clin Invest. 2005;115:1111-1119.

9. Navarro JF, Mora C. Role of inflammation in diabetic complications. Nephrol Dial Transplant. 2005;20:2601-2604.

10. DeFronzo RA. From the triumvirate to the ominous Octet: a new paradigm for the treatment of type 2 diabetes mellitus. Diabetes. 2009;58:773-795.

11. Fonseca VA. Defining and characterizing the progression of type 2 diabetes. Diabetes Care. 2009;32:S151-S156.

12. Bloomgarden ZT, Handelsman Y. Approaches to treatment 2: comparison of AACE and ADA type 2 diabetes treatment guidelines. J Diabetes. 2015;8(1):4-6.

13. Shoelson SE, Lee J, Goldfine AB. Inflammation and insulin resistance. J Clin Invest. 2006;116:1793-1801.

14. Hotamisligil GS, Shargill NS, Spiegelman BM. Adipose expression of tumor necrosis factor-alpha: direct role in obesity-linked insulin resistance. Science. 1993;259:87-91.

15. Pickup JC, Crook MA. Is type II diabetes mellitus a disease of the innate immune system? Diabetologia. 1998;41:1241-1248.

16. Spranger J, Kroke A, Möhlig M, et al. Inflammatory cytokines and the risk to develop type 2 diabetes: results of the prospective populationbased European Prospective Investigation into Cancer and Nutrition (EPIC)-Potsdam Study. Diabetes. 2003;52:812-817.

17. King GL. The role of inflammatory cytokines in diabetes and its complications. J Periodontol. 2008;79:1527-1534.

18. Surmi BK, Hasty AH. Macrophage infiltration into adipose tissue: initiation, propagation and remodeling. Future Lipidol. 2008;3:545-556.

19. Lumeng CN, Bodzin JL, Saltiel AR. Obesity induces a phenotypic switch in adipose tissue macrophage polarization. J Clin Invest. 2007;117:175-184.

20. Cernea S, Dobreanu M. Diabetes and beta cell function: from mechanisms to evaluation and clinical implications. Biochem Med (Zagreb). 2013;23:266-280.

21. Sell H, Habich C, Eckel J. Adaptive immunity in obesity and insulin resistance. Nat Rev Endocrinol. 2012;8:709-716.

22. Dandona P, Chaudhuri A, Ghanim H, et al. Insulin as an antiinflammatory and antiatherogenic modulator. J Am Coll Cardiol. 2009;53:S14-S20.

23. Dandona P, Chaudhuri A. Anti-inflammatory and antiatherogenic effects of insulin. Insulin. 2006;1 Suppl 1:S11-S17.

24. Ng KW, Allen ML, Desai A, et al. Cardioprotective effects of insulin: how intensive insulin therapy may benefit cardiac surgery patients. Circulation. 2012;125:721-728.

25. Mao XM, Liu H, Tao XJ, et al. Independent anti-inflammatory effect of insulin in newly diagnosed type 2 diabetes. Diabetes Metab Res Rev. 2009;25:435-441.

26. Ghanim H, Mohanty P, Deopurkar R, et al. Acute modulation of toll-like receptors by insulin. Diabetes Care. 2008;31:1827-1831. 
27. Niswender K. Early and aggressive initiation of insulin therapy for type 2 diabetes: what is the evidence? Clin Diabetes. 2009;27:60-68.

28. Meneghini LF. Early insulin treatment in type 2 diabetes. Diabetes Care. 2009;32:S266-S269.

29. The Action to Control Cardiovascular Risk in Diabetes Study Group. Effects of intensive glucose lowering in type 2 diabetes. $N$ Engl J Med. 2008;358:2545-2559.

30. Takebayashi $\mathrm{K}$, Aso $\mathrm{Y}$, Inukai $\mathrm{T}$. Initiation of insulin therapy reduces serum concentrations of high-sensitivity C-reactive protein in patients with type 2 diabetes. Metab Clin Exp. 2004;53:693-699.

31. Da Silva AMV, de Moraes Penno L, Bertoluci MC, et al. Insulin therapy does not interfere with venous endothelial function evaluation in patients with type 2 diabetes mellitus. Clinics (Sao Paulo). 2010;65:1139-1142.

32. Pfützner A, SchöndorfT, Hanefeld M, et al. High-sensitivity C-reactive protein predicts cardiovascular risk in diabetic and nondiabetic patients: effects of insulin-sensitizing treatment with pioglitazone. $J$ Diabetes Sci Technol. 2010;4:706-716.

33. Pradhan AD, Everett BM, Cook NR, et al. Effects of initiating insulin and metformin on glycemic control and inflammatory biomarkers among patients with type 2 diabetes: the LANCET randomized trial. JAMA. 2009;302:1186-1194.

34. Jansen HJ, Stienstra R, van Diepen JA, et al. Start of insulin therapy in patients with type 2 diabetes mellitus promotes the influx of macrophages into subcutaneous adipose tissue. Diabetologia. 2013;56: 2573-2581.

35. Galdo JA, Thurston MM, Bourg CA. Clinical considerations for insulin pharmacotherapy in ambulatory care, part one: introduction and review of current products and guidelines. Clin Diabetes. 2014;32:66-75.

36. Wang XC, Gusdon AM, Liu H, et al. Effects of glucagon-like peptide-1 receptor agonists on non-alcoholic fatty liver disease and inflammation. World J Gastroenterol. 2014;20:14821-14830.

37. Avogaro A, Vigili de Kreutzenberg S, Fadini GP. Cardiovascular actions of GLP-1 and incretin-based pharmacotherapy. Curr Diab Rep. 2014; $14: 483$.

38. Pi-Sunyer X, Astrup A, Fujioka K, et al. A randomized, controlled trial of $3.0 \mathrm{mg}$ of liraglutide in weight management. $N$ Engl $J$ Med. $2015 ; 373: 11-22$.

39. Liu J, Wang G, Jia Y, et al. GLP-1 receptor agonists: effects on the progression of non-alcoholic fatty liver disease. Diabetes Metab Res Rev. 2015;31:329-335.

40. Lee YS, Park MS, Choung JS, et al. Glucagon-like peptide-1 inhibits adipose tissue macrophage infiltration and inflammation in an obese mouse model of diabetes. Diabetologia. 2012;55:2456-2468.

41. Saisho Y. Metformin and inflammation: its potential beyond glucoselowering effect. Endocr Metab Immune Disord Drug Targets. 2015;15: 196-205.

42. Isoda K, Young JL, Zirlik A, et al. Metformin inhibits proinflammatory responses and nuclear factor-kappaB in human vascular wall cells. Arterioscler Thromb Vasc Biol. 2006;26:611-617.

43. Kim SA, Choi HC. Metformin inhibits inflammatory response via AMPK-PTEN pathway in vascular smooth muscle cells. Biochem Biophys Res Commun. 2012;425:866-872.

44. Zheng Z, Chen H, Li J, et al. Sirtuin 1-mediated cellular metabolic memory of high glucose via the LKB1/AMPK/ROS pathway and therapeutic effects of metformin. Diabetes. 2012;61:217-228.

45. Ruggiero-Lopez D, Lecomte M, Moinet G, et al. Reaction of metformin with dicarbonyl compounds. Possible implication in the inhibition of advanced glycation end product formation. Biochem Pharmacol. 1999;58:1765-1773.

46. Haffner S, Temprosa M, Crandall J, et al. Intensive lifestyle intervention or metformin on inflammation and coagulation in participants with impaired glucose tolerance. Diabetes. 2005;54:1566-1572.

47. Krysiak R, Okopien B. The effect of metformin on monocyte secretory function in simvastatin-treated patients with impaired fasting glucose. Metab Clin Exp. 2013;62:39-43.
48. Krysiak R, Okopien B. Lymphocyte-suppressing and systemic anti-inflammatory effects of high-dose metformin in simvastatintreated patients with impaired fasting glucose. Atherosclerosis. 2012;225:403-407.

49. Sobel BE, Hardison RM, Genuth S, et al. Profibrinolytic, antithrombotic, and antiinflammatory effects of an insulin-sensitizing strategy in patients in the Bypass Angioplasty Revascularization Investigation 2 Diabetes (BARI 2D) trial. Circulation. 2011;124:695-703.

50. Derosa G, Carbone A, D'Angelo A, et al. Variations in inflammatory biomarkers following the addition of sitagliptin in patients with type 2 diabetes not controlled with metformin. Intern Med. 2013;52:2179-2187.

51. Proks P, Reimann F, Green N, et al. Sulfonylurea stimulation of insulin secretion. Diabetes. 2002;51 Suppl 3:S368-S376.

52. Ling MY, Ma ZY, Wang YY, et al. Up-regulated ATP-sensitive potassium channels play a role in increased inflammation and plaque vulnerability in macrophages. Atherosclerosis. 2013;226:348-355.

53. Cai J, Lu S, Yao Z, Deng YP, et al. Glibenclamide attenuates myocardial injury by lipopolysaccharides in streptozotocin-induced diabetic mice. Cardiovasc Diabetol. 2014;13:106.

54. Mu-Huo J, Jiao-Jiao Y, Lin-Sha J, et al. Glibenclamide pretreatment attenuates acute lung injury by inhibiting the inflammatory responses and oxidative stress in a polymicrobial sepsis animal model. JAPM. 2014;1:36-43

55. Simard JM, Geng Z, Woo SK, et al. Glibenclamide reduces inflammation, vasogenic edema, and caspase-3 activation after subarachnoid hemorrhage. J Cereb Blood Flow Metab. 2009;29:317-330.

56. Kewcharoenwong $C$, Rinchai $\mathrm{D}$, Utispan $\mathrm{K}$, et al. Glibenclamide reduces pro-inflammatory cytokine production by neutrophils of diabetes patients in response to bacterial infection. Sci Rep. 2013;3:3363.

57. Mavridis G, Souliou E, Diza E, et al. Inflammatory cytokines in insulin-treated patients with type 2 diabetes. Nutr Metab Cardiovasc Dis. 2008;18:471-476.

58. Cui W, Zhang S, Cai Z, et al. The antidiabetic agent glibenclamide protects airway hyperresponsiveness and inflammation in mice. Inflammation. 2015;38:835-845.

59. Derosa G, D'Angelo A, Salvadeo SAT, et al. Modification of vascular and inflammation biomarkers after OGTT in overweight healthy and diabetic subjects. Microvasc Res. 2010;79:144-149.

60. Derosa G, D'Angelo A, Salvadeo SAT, et al. Oral glucose tolerance test effects on endothelial inflammation markers in healthy subjects and diabetic patients. Horm Metab Res. 2010;42:8-13.

61. Fukaya N, Mochizuki K, Shimada M, et al. The alpha-glucosidase inhibitor miglitol decreases glucose fluctuations and gene expression of inflammatory cytokines induced by hyperglycemia in peripheral leukocytes. Nutrition. 2009;25:657-667.

62. Derosa G, Maffioli P, D'Angelo A, et al. Acarbose on insulin resistance after an oral fat load: a double-blind, placebo controlled study. $J$ Diabetes Complications. 2011;25:258-266.

63. Derosa G, Maffioli P, Ferrari I, et al. Acarbose actions on insulin resistance and inflammatory parameters during an oral fat load. Eur $J$ Pharmacol. 2011;651:240-250.

64. Emoto T, Sawada T, Hashimoto M, et al. Effect of 3-month repeated administration of miglitol on vascular endothelial function in patients with diabetes mellitus and coronary artery disease. Am J Cardiol. 2012;109:42-46.

65. Shimazu T, Inami N, Satoh D, et al. Effect of acarbose on plateletderived microparticles, soluble selectins, and adiponectin in diabetic patients. J Thromb Thrombolysis. 2009;28:429-435.

66. Fujitaka K, Otani H, Jo F, et al. Comparison of metabolic profile and adiponectin level with pioglitazone versus voglibose in patients with type-2 diabetes mellitus associated with metabolic syndrome. Endocr $J$. 2011;58:425-432.

67. Tung D, Cheung PH, Ciallella J, et al. Novel anti-inflammatory effects of repaglinide in rodent models of inflammation. Pharmacology. 2011;88:295-301. 
68. Yamazaki M, Hasegawa G, Majima S, et al. Effect of repaglinide versus glimepiride on daily blood glucose variability and changes in blood inflammatory and oxidative stress markers. Diabetol Metab Syndr. 2014;6:54

69. Assaloni R, Da Ros R, Quagliaro L, et al. Effects of S21403 (mitiglinide) on postprandial generation of oxidative stress and inflammation in type 2 diabetic patients. Diabetologia. 2005;48:1919-1924.

70. Gumieniczek A, Hopkała H, Roliński J, et al. Antioxidative and antiinflammatory effects of repaglinide in plasma of diabetic animals Pharmacol Res. 2005;52:162-166.

71. Lund SS, Tarnow L, Stehouwer CDA, et al. Impact of metformin versus repaglinide on non-glycaemic cardiovascular risk markers related to inflammation and endothelial dysfunction in non-obese patients with type 2 diabetes. Eur J Endocrinol. 2008;158:631-641.

72. Holman RR, Haffner SM, McMurray JJ, et al. Effect of nateglinide on the incidence of diabetes and cardiovascular events. $N$ Engl J Med. 2010;362:1463-1476.

73. Ju-Ming L, Xiao-Hui G, Xiao-Feng L, et al. Effects of nateglinide on postprandial plasma glucose excursion and metabolism of lipids in Chinese patients with type 2 diabetes: a 4-week, randomized, activecontrol, open-label, parallel-group, multicenter trial. Curr Med Res Opin. Epub 2012 Jul 19.

74. Corzo C, Griffin PR. Targeting the peroxisome proliferator-activated receptor $\gamma$ to counter the inflammatory milieu in obesity. Diabetes Metab J. 2013;37:395-403.

75. Ialenti A, Grassia G, Di Meglio P, et al. Mechanism of the antiinflammatory effect of thiazolidinediones: relationship with the glucocorticoid pathway. Mol Pharmacol. 2005;67:1620-1628.

76. Koppaka S, Kehlenbrink S, Carey M, et al. Reduced adipose tissue macrophage content is associated with improved insulin sensitivity in thiazolidinedione-treated diabetic humans. Diabetes. 2013;62: 1843-1854.

77. Szanto A, Nagy L. The many faces of PPARgamma: anti-inflammatory by any means? Immunobiology. 2008;213:789-803.

78. Powell LA, Crowe P, Kankara C, et al. Restoration of adipose function in obese glucose-tolerant men following pioglitazone treatment is associated with CCAAT enhancer-binding protein $\beta$ up-regulation. Clin Sci. 2012;123:135-146.

79. Esterson YB, Zhang K, Koppaka S, et al. Insulin sensitizing and antiinflammatory effects of thiazolidinediones are heightened in obese patients. J Investig Med. 2013;61:1152-1160.

80. Kodama N, Tahara N, Tahara A, et al. Effects of pioglitazone on visceral fat metabolic activity in impaired glucose tolerance or type 2 diabetes mellitus. J Clin Endocrinol Metab. 2013;98:4438-4445.

81. Cipolletta D, Feuerer M, Li A, et al. PPAR- $\gamma$ is a major driver of the accumulation and phenotype of adipose tissue Treg cells. Nature. 2012;486:549-553.

82. Shyangdan D, Clar C, Ghouri N, et al. Insulin sensitisers in the treatment of non-alcoholic fatty liver disease: a systematic review. Health Technol Assess. 2011;15:1-110.

83. Boettcher E, Csako G, Pucino F, et al. Meta-analysis: pioglitazone improves liver histology and fibrosis in patients with non-alcoholic steatohepatitis. Aliment Pharmacol Ther. 2012;35:66-75.

84. Plutzky J. The potential role of peroxisome proliferator-activated receptors on inflammation in type 2 diabetes mellitus and atherosclerosis. Am J Cardiol. 2003;92:34J-41J.

85. Nitta Y, Tahara N, Tahara A, et al. Pioglitazone decreases coronary artery inflammation in impaired glucose tolerance and diabetes mellitus: evaluation by FDG-PET/CT imaging. JACC Cardiovasc Imaging 2013;6:1172-1182.

86. Mizoguchi M, Tahara N, Tahara A, et al. Pioglitazone attenuates atherosclerotic plaque inflammation in patients with impaired glucose tolerance or diabetes a prospective, randomized, comparatorcontrolled study using serial FDG PET/CT imaging study of carotid artery and ascending aorta. JACC Cardiovasc Imaging. 2011;4: 1110-1118.
87. Marfella R, D’Amico M, Esposito K, et al. The ubiquitin-proteasome system and inflammatory activity in diabetic atherosclerotic plaques: effects of rosiglitazone treatment. Diabetes. 2006;55:622-632.

88. Nissen SE, Nicholls SJ, Wolski K, et al. Comparison of pioglitazone vs glimepiride on progression of coronary atherosclerosis in patients with type 2 diabetes: the PERISCOPE randomized controlled trial. JAMA. 2008;299:1561-1573.

89. Chen R, Yan J, Liu P, Wang Z. Effects of thiazolidinedione therapy on inflammatory markers of type 2 diabetes: a meta-analysis of randomized controlled trials. PLoS ONE. 2015;10:e0123703.

90. Zhao Y, He X, Huang C, et al. The impacts of thiazolidinediones on circulating C-reactive protein levels in different diseases: a metaanalysis. Diabetes Res Clin Pract. 2010;90:279-287.

91. Karagiannis E, Pfützner A, Forst T, et al. The IRIS V study: pioglitazone improves systemic chronic inflammation in patients with type 2 diabetes under daily routine conditions. Diabetes Technol Ther. 2008;10:206-212.

92. Dormandy JA, Charbonnel B, Eckland DJA, et al. Secondary prevention of macrovascular events in patients with type 2 diabetes in the PROactive Study (PROspective pioglitAzone Clinical Trial In macroVascular Events): a randomised controlled trial. Lancet. 2005;366:1279-1289.

93. Ussher JR, Drucker DJ. Cardiovascular biology of the incretin system. Endocr Rev. 2012;33:187-215.

94. Scheen AJ. Cardiovascular effects of gliptins. Nat Rev Cardiol. 2013;10:73-84.

95. Zhao Y, Yang L, Zhou Z. Dipeptidyl peptidase-4 inhibitors: multitarget drugs, not only antidiabetes drugs. J Diabetes. 2014;6:21-29.

96. Avogaro A, Fadini GP. The effects of dipeptidyl peptidase-4 inhibition on microvascular diabetes complications. Diabetes Care. 2014;37:2884-2894.

97. Hirakawa H, Zempo H, Ogawa M, et al. A DPP-4 inhibitor suppresses fibrosis and inflammation on experimental autoimmune myocarditis in mice. PLoS ONE. 2015;10:e0119360.

98. Schürmann C, Linke A, Engelmann-Pilger K, et al. The dipeptidyl peptidase-4 inhibitor linagliptin attenuates inflammation and accelerates epithelialization in wounds of diabetic ob/ob mice. J Pharmacol Exp Ther. 2012;342:71-80.

99. Ta NN, Schuyler CA, Li Y, et al. DPP-4 (CD26) inhibitor alogliptin inhibits atherosclerosis in diabetic apolipoprotein E-deficient mice. J Cardiovasc Pharmacol. 2011;58:157-166.

100. Dai Y, Dai D, Wang X, et al. DPP-4 inhibitors repress NLRP3 inflammasome and interleukin-1beta via GLP-1 receptor in macrophages through protein kinase C pathway. Cardiovasc Drugs Ther. 2014;28:425-432.

101. Shinjo T, Nakatsu Y, Iwashita M, et al. DPP-IV inhibitor anagliptin exerts anti-inflammatory effects on macrophages, adipocytes, and mouse livers by suppressing NF- $\kappa \mathrm{B}$ activation. Am J Physiol Endocrinol Metab. 2015;309:E214-E223.

102. Makdissi A, Ghanim H, Vora M, et al. Sitagliptin exerts an antiinflammatory action. J Clin Endocrinol Metab. 2012;97:3333-3341.

103. Tremblay AJ, Lamarche B, Deacon CF, et al. Effects of sitagliptin therapy on markers of low-grade inflammation and cell adhesion molecules in patients with type 2 diabetes. Metab Clin Exp 2014;63:1141-1148.

104. Klempfner R, Leor J, Tenenbaum A, et al. Effects of a vildagliptin/ metformin combination on markers of atherosclerosis, thrombosis, and inflammation in diabetic patients with coronary artery disease. Cardiovasc Diabetol. 2012;11:60.

105. Satoh-Asahara N, Sasaki Y, Wada H, et al. A dipeptidyl peptidase-4 inhibitor, sitagliptin, exerts anti-inflammatory effects in type 2 diabetic patients. Metab Clin Exp. 2013;62:347-351.

106. Matsubara J, Sugiyama S, Akiyama E, et al. Dipeptidyl peptidase-4 inhibitor, sitagliptin, improves endothelial dysfunction in association with its anti-inflammatory effects in patients with coronary artery disease and uncontrolled diabetes. Circ J. 2013;77:1337-1344. 
107. Derosa G, Maffioli P, Salvadeo SAT, et al. Effects of sitagliptin or metformin added to pioglitazone monotherapy in poorly controlled type 2 diabetes mellitus patients. Metab Clin Exp. 2010;59: 887-895.

108. Nakamura Y, Tsuji M, Hasegawa H, et al. Anti-inflammatory effects of linagliptin in hemodialysis patients with diabetes. Hemodial Int. 2014; $18: 433-442$.

109. Scheen AJ. Pharmacodynamics, efficacy and safety of sodium-glucose co-transporter type 2 (SGLT2) inhibitors for the treatment of type 2 diabetes mellitus. Drugs. 2015;75:33-59.

110. Tahara A, Kurosaki E, Yokono M, et al. Effects of sodium-glucose cotransporter 2 selective inhibitor ipragliflozin on hyperglycaemia, oxidative stress, inflammation and liver injury in streptozotocin-induced type 1 diabetic rats. J Pharm Pharmacol. 2014;66:975-987.

111. Tahara A, Kurosaki E, Yokono M, et al. Effects of SGLT2 selective inhibitor ipragliflozin on hyperglycemia, hyperlipidemia, hepatic steatosis, oxidative stress, inflammation, and obesity in type 2 diabetic mice. Eur J Pharmacol. 2013;715:246-255.

112. Hermansen K, Mortensen LS, Hermansen M-L. Combining insulins with oral antidiabetic agents: effect on hyperglycemic control, markers of cardiovascular risk and disease. Vasc Health Risk Manag. 2008;4:561-574

113. Schöndorf T, Musholt PB, Hohberg C, et al. The fixed combination of pioglitazone and metformin improves biomarkers of platelet function and chronic inflammation in type 2 diabetes patients: results from the PIOfix study. J Diabetes Sci Technol. 2011;5:426-432.

114. Hanefeld M. Pioglitazone and sulfonylureas: effectively treating type 2 diabetes. Int J Clin Pract Suppl. 2007;61 Suppl 153:20-27.

115. Derosa G, Franzetti IG, Querci F, et al. Variation in inflammatory markers and glycemic parameters after 12 months of exenatide plus metformin treatment compared with metformin alone: a randomized placebo-controlled trial. Pharmacotherapy. 2013;33:817-826.
116. Chen L, Klein T, Leung PS. Effects of combining linagliptin treatment with BI-38335, a novel SGLT2 inhibitor, on pancreatic islet function and inflammation in $\mathrm{db} / \mathrm{db}$ mice. Curr Mol Med. 2012;12:995-1004.

117. Nauck MA. Update on developments with SGLT2 inhibitors in the management of type 2 diabetes. Drug Des Dev Ther. 2014;8: 1335-1380.

118. Grover JK, Yadav S. Vats V. Medicinal plants of India with anti-diabetic potential. J Ethnopharmacol 2002; 81:81-100.

119. Grover JK, Vats V, Yadav S. Effect of feeding aqueous extract of Petrocarpus marsupium on glycogen content of tissues and the key enzymes of carbohydrate metabolism. Molecular and Cellular Biochemistry. 2002; 241:53-59.

120. Babu PVA, Liu D. Green tea catechins and cardiovascular health: an update. Curr Med Chem. 2008;15:1840-1850.

121. Farrar MD, Nicolaou A, Clarke KA, et al. A randomized controlled trial of green tea catechins in protection against ultraviolet radiationinduced cutaneous inflammation. Am J Clin Nutr. 2015;102:608-615.

122. Satti NK, Chauhan PS, Dutt P, et al. Amelioration of inflammatory responses by chlorogenic acid via suppression of pro-inflammatory mediators. J Appl Pharm Sci. 2011;1:67-75.

123. Shin HS, Satsu H, Bae MJ, et al. Anti-inflammatory effect of chlorogenic acid on the IL- 8 production in Caco- 2 cells and the dextran sulphate sodium-induced colitis symptoms in C57BL/6 mice. Food Chem. 2015;168:167-175.

124. González R, Ballester I, López-Posadas R, et al. Effects of flavonoids and other polyphenols on inflammation. Crit Rev Food Sci Nutr. 2011;51:331-362.

125. Lee Y, Jung JC, Ali Z, Khan IA, Oh S. Anti-inflammatory effect of triterpene saponins isolated from blue cohosh (caulophyllum thalictroides). Evid Based Complement Alternat Med. 2012;2012:798192.
Journal of Inflammation Research

\section{Publish your work in this journal}

The Journal of Inflammation Research is an international, peer-reviewed open-access journal that welcomes laboratory and clinical findings on the molecular basis, cell biology and pharmacology of inflammation including original research, reviews, symposium reports, hypothesis formation and commentaries on: acute/chronic inflammation; mediators of inflamma-

\section{Dovepress}

tion; cellular processes; molecular mechanisms; pharmacology and novel anti-inflammatory drugs; clinical conditions involving inflammation. The manuscript management system is completely online and includes a very quick and fair peer-review system. Visit http://www.dovepress.com/ testimonials.php to read real quotes from published authors. 